\title{
Neutralising Antibodies against Enterovirus and Parechovirus in IVIG Reflect General Circulation: A Tool for Sero-Surveillance
}

\author{
Karen Couderé ${ }^{1, *}$, Karlijn van der Straten ${ }^{2}$ (D) Lieke Brouwer $^{2}$, Gerrit Koen ${ }^{2}$, Hetty van Eijk ${ }^{2}$, Dasja Pajkrt ${ }^{3}$, , \\ Jean-Luc Murk ${ }^{1}$ and Katja C. Wolthers ${ }^{2}$ (D)
}

1 Department of Medical Microbiology Microvida, Elisabeth-Tweesteden Ziekenhuis, 5022 GC Tilburg, The Netherlands; j.murk@etz.nl

2 Department of Medical Microbiology, Amsterdam University Medical Centres, Locatie AMC, 1105 AZ Amsterdam, The Netherlands; k.vanderstraten@amsterdamumc.nl (K.v.d.S.);

liekebrouwer@hotmail.com (L.B.); g.koen@amsterdamumc.nl (G.K.); h.w.vaneijk@amsterdamumc.nl (H.v.E.); k.c.wolthers@amsterdamumc.nl (K.C.W.)

3 Emma Children's Hospital, Department of Pediatric Infectious Diseases, Amsterdam University Medical Centres, Location AMC, 1105 AZ Amsterdam, The Netherlands; d.pajkr@@amsterdamumc.nl

* Correspondence: k.coudere@etz.nl; Tel.: +32-497-92-49-62

Citation: Couderé, K.; van der Straten, K.; Brouwer, L.; Koen, G.; van Eijk, H.; Pajkrt, D.; Murk, J.-L.; Wolthers, K.C. Neutralising Antibodies against Enterovirus and Parechovirus in IVIG Reflect General Circulation: A Tool for Sero-Surveillance. Viruses 2021, 13, 1028. https://doi.org/10.3390/ v13061028

Academic Editors: Javier Martin and Erwin Duizer

Received: 23 April 2021

Accepted: 25 May 2021

Published: 29 May 2021

Publisher's Note: MDPI stays neutral with regard to jurisdictional claims in published maps and institutional affiliations.

Copyright: (c) 2021 by the authors. Licensee MDPI, Basel, Switzerland. This article is an open access article distributed under the terms and conditions of the Creative Commons Attribution (CC BY) license (https:/ / creativecommons.org/licenses/by/ $4.0 /)$.

\begin{abstract}
Non-polio enteroviruses (NPEV) and parechoviruses (PeV) are widespread pathogens that cause significant morbidity. Surveillance is based on culturing or genotyping of virus strains found in clinical samples. Sero-surveillance, by measuring neutralising antibodies ( $\mathrm{nAb}$ ) through virus neutralisation assays (VNA), could provide additional information as it offers a more comprehensive overview of exposure to circulating types in the general population. In our study we evaluated Intravenous immunoglobulins (IVIG) to generate sero-surveillance data. We performed VNA of nineteen NPEV and PeV with Dutch IVIG batches from two different time points (2010 and 2017) and an IVIG batch from Vietnam (2011). We compared our findings with geno- and sero-surveillance data and evaluated changes over time and between the two countries. Our findings show a good correlation with what is known from geno-surveillance data. The highest $\mathrm{nAb}$ titres were found against strains from Enterovirus B, while we did not observe $\mathrm{nAb}$ titres against strains belonging to Enterovirus $C$. In conclusion, we demonstrated that sero-surveillance by means of IVIG can be used to obtain insight into circulation of $\mathrm{EV}$ and $\mathrm{PeV}$ genotypes. This is of particular interest for public health, to evaluate changes over time and population susceptibility to emerging genotypes.
\end{abstract}

Keywords: Enterovirus; Parechovirus; surveillance; sero-surveillance; virus neutralisation assay

\section{Introduction}

Numerous enteroviruses $(\mathrm{EV})$ and parechoviruses $(\mathrm{PeV})$ have recently caused large outbreaks, including EV-A71, EV-D68 and PeV-3 [1,2]. These viruses belong to the Picornaviridae family, one of the largest RNA virus families. Human EV and PeV cause a wide range of disease from mild upper respiratory infections to more serious infections including hand-foot-mouth disease (HFMD), myocarditis, neonatal sepsis and neurological disease [3]. They are the most common causative pathogen of viral meningitis $[4,5]$ and some types are known to cause (meningo-) encephalitis, brainstem encephalitis and acute flaccid myelitis / paralysis (AFM/AFP) causing important morbidity and mortality worldwide. Human EV contain more than 100 different types and are subdivided into four species; Enterovirus $A$ to Enterovirus D, which include coxsackie (CV) A- and B-, echoand numbered enteroviruses (EV). Human PeV, of which PeV-1 and PeV-2 were originally misclassified as E22 and E23, belong to Parechovirus A species, which currently contains 19 types: PeV-A1 up to PeV-A19. Classification of EV and PeV is based on molecular 
typing which is performed by partial sequencing of the viral capsid protein 1 (VP1) region. These genotypes correlate largely with previously defined serotypes as this region contains most of the neutralising epitopes [2]. The term non-polio enteroviruses (NPEV) refers to all enterovirus types except for the three poliovirus types. Despite the serious morbidity NPEV and PeV can cause, many countries do not have systematic surveillance in place and knowledge on circulating types is mainly based on genotyping through VP1 sequencing of $\mathrm{EV}$ and $\mathrm{PeV}$ found in clinical samples. Although it provides an important insight in the current types causing serious disease which require hospitalization, a disadvantage of this method is that it does not represent all circulating virus types in the population. Sero-surveillance, by detecting neutralising antibodies $(\mathrm{nAb})$ against specific virus types through Virus Neutralisation Assays (VNA), reflects the magnitude at which a population has been in contact with certain virus types, including those causing mild or no disease and emerging subtypes [6]. This information, together with genotyping data, could help to predict circulation patterns [7]. Moreover, during outbreaks, it has the potential to provide information on the level of protection of a population against the outbreak strain. Serosurveillance has several challenges however, like requiring access to large representative serum banks. Intravenous immunoglobulins (IVIG) provide the unique opportunity to investigate a representative sample from the general population as it contains plasma from at least 1000 healthy adult donors from different age groups $[6,8]$. In our study we evaluated IVIG as a specimen, representative of the population, to generate sero-surveillance data for the most commonly detected NPEV and PeV in the Netherlands. We analysed changes over time by comparing two Dutch IVIG batches from 2010 and 2017, and differences between geographical regions by comparing with a 2011 IVIG batch from Vietnam. The results were related to geno- and sero-surveillance data from the literature.

\section{Materials and Methods}

\subsection{IVIG Selection}

Three IVIG batches were selected; two from the Netherlands and one from Vietnam. The Dutch IVIG batches (Nanogam, Sanquin, the Netherlands) were fabricated in 2010 and 2017. The Vietnamese IVIG batch (Green Cross Corporation, Pymepharco, Vietnam) was fabricated in 2011 and kindly provided by R. van Doorn from the Ho Chi Minh Hospital for Tropical Diseases. The protein concentrations from the Dutch 2010, 2017 and Vietnamese 2011 IVIG batches were, respectively $49.9 \mathrm{mg} / \mathrm{mL}, 50.6 \mathrm{mg} / \mathrm{mL}$ and $55.1 \mathrm{mg} / \mathrm{mL}$. As a positive control, we used PeV-A1 (Harris B) as we had previously detected high nAb titres against this strain in Dutch IVIG [9]. The positive control was neutralised by all three IVIG batches.

\subsection{Selection of NPEV and PeV strains}

The selection of prevalent circulating types was based on VIRO-TypeNed data. In the Netherlands this web-based platform is used for surveillance of EV and PeV with collection of demographic, clinical and sequence-typing data from laboratories countrywide on a voluntary basis [10]. We defined a highly prevalent type as being listed more than once in the top five most prevalent viruses circulating between 2011 and 2017 (Table 1). This resulted in the inclusion of the following twelve virus types: CVA6, E6, E9, E11, E18, E25, E30, CVB5, CVA9, PeV-A1, PeV-A3, PeV-A4. Additionally, three types were included based on association with disease: one EV-D68 strain, two EV-A71 strains and one CVA16 strain. Additionally, four types from Enterovirus $C$ species, which rarely occur in the VIRO-TypeNed surveillance data, were included: CVA11, CVA13, CVA20 and CVA24. For each type, we selected the most recent isolated clinical strain from the Laboratory of Medical Microbiology Amsterdam University Medical Centres (Amsterdam UMC) location AMC, to match the current circulating strains. CVA11 was kindly provided by the Leiden University Medical Centre (LUMC). CVA13, CVA20, CVA24 and the EV-D68 strain were kindly provided by the National Institute of Public Health and Environment (RIVM). The EV-A71C4 strain from Japan was kindly provided by K. Mizuta from the Yamagata 
Prefectural Institute of Public Health. Characteristics of the selected strains can be found in Supplementary Table S1.

Table 1. VIRO-Typened top five most prevalent viruses circulating between 2010 and 2017 in the Netherlands (adapted from Benschop 2016, source VIRO-TypeNed). EV; enterovirus, PeV; parechovirus, CVA; coxsackievirus A, CVB; coxsackievirus B, E; echovirus.

\begin{tabular}{ccccccccc}
\hline Ranking & $\mathbf{2 0 1 0}$ & $\mathbf{2 0 1 1}$ & $\mathbf{2 0 1 2}$ & $\mathbf{2 0 1 3}$ & $\mathbf{2 0 1 4}$ & $\mathbf{2 0 1 5}$ & $\mathbf{2 0 1 6}$ & $\mathbf{2 0 1 7}$ \\
\hline $\mathbf{1}$ & CVA9 & E-25 & E-18 & CVB3 & E-16 & E-11 & E-30 & E-5 \\
\hline $\mathbf{2}$ & EV-A71 & E-7 & CVA6 & CVA9 & E-25 & CVA6 & EV-D68 & CVB5 \\
\hline $\mathbf{3}$ & PeV-A1 & PeV-A1 & PeV-A1 & E-30 & CVA6 & E-9 & E-6 & CVA6 \\
\hline $\mathbf{4}$ & E-30 & CVB3 & PeV-A3 & EV-A71 & CVA16 & E-18 & CVB5 & E-25 \\
\hline $\mathbf{5}$ & PeV-A3 & CVB4 & E-9 & PeV-A1 & EV-D68 & CVB5 & PeV-A3 & E-9 \\
\hline
\end{tabular}

\subsection{Cell Line Selection}

All strains were cultured on six different cell lines: Human Colorectal Adenocarcinoma (HT29), African Green Monkey Kidney (Vero), Rhabdomyosarcoma (RD), Rhesus Monkey Kidney Epithelial (LLC-MK2), Human Embryo Lung (HEL) and Human Lung Adenocarcinoma (A549). Cells were cultured at $37^{\circ} \mathrm{C} 5 \%$ carbon dioxide $\left(\mathrm{CO}_{2}\right)$ in Eagles minimum essential medium (EMEM, Lonza, Switzerland) supplemented with $8 \%$ foetal bovine serum (FBS, Sigma-Aldrich, United States), $100 \mathrm{IU} / \mathrm{mL}$ penicillin and $100 \mu \mathrm{g} / \mathrm{mL}$ streptomycin. We selected the most appropriate cell line for each viral strain, that is with a cytopathological effect $\geq 50 \%$ within 7 days, to perform the serum neutralisation assay. HT cells were selected for most viruses (9/19), followed by RD (6/19), Hel (3/19) and LLC-MK2 (1/19). CVA24 did not grow on any of the cell lines and therefore had to be excluded from further experiments.

\subsection{Dilution Neutralisation Assay}

Neutralising antibody (nAb) titres of IVIG batches against the selected viruses were determined by using a virus neutralisation assay. An in-duplicate 2 -fold serial dilution of the IVIG batches were incubated with an equal volume of $100 \mu \mathrm{L} \mathrm{50 \%} \mathrm{tissue} \mathrm{culture}$ infective dose $\left(\mathrm{TCID}_{50}\right)$ of virus at $37^{\circ} \mathrm{C}$ in $5 \% \mathrm{CO}_{2}$ for one hour. PeV-3 and virus strains that tested negative in the first neutralisation assay (EV-A71C1, CVA16, E18, CVA11, CVA13 and CVA20) were pre-treated with chloroform to overcome virus aggregation and optimize neutralisation [11,12]. HT29, RD, Hel, Vero or LLC-MK2 cells in EMEM supplemented with $8 \%$ FBS were subsequently added and incubated for 7 days at $37{ }^{\circ} \mathrm{C} 5 \% \mathrm{CO}_{2}$. We determined $\mathrm{nAb}$ titres based on the cytopathologic effect using the Reed and Muench method. We reported $\mathrm{nAb}$ titres as the reciprocal titres of serum dilutions exhibiting $50 \%$ neutralisation. Titres of $\geq 1: 16$ and $\geq 1: 32$ were defined as seropositive and protective, respectively. We divided $\mathrm{nAb}$ titres based on their level: absence of titres $(<1: 16)$, low titres (1:16-1:128), medium titres (1:129-1:511) and high titres (1:512-1:2048) [13,14].

\subsection{Statistical Analysis}

Statistics were performed in $\mathrm{R}$ ( $\mathrm{R}$ core team 2020). We used the Kruskal-Wallis test to compare $\mathrm{nAb}$ titres between the different species and the Mann-Whitney-Wilcoxon test to compare $\mathrm{nAb}$ titres between IVIG batches for the different species. $p<0.05$ was defined as significant. A titre difference of more than 2-fold between $\mathrm{nAb}$ against two individual types was deemed relevant, while a difference of 2-fold or less was determined as inter-assay variability. 


\section{Results}

A total of 19 virus types were included: 3 Parechovirus $A, 4$ Enterovirus $A, 8$ Enterovirus $B, 3$ Enterovirus $C$ and 1 Enterovirus $D$ type. The $\mathrm{nAb}$ titres from the different IVIG batches against the selected viral strains are presented in Figure 1 and Supplementary Table S2.
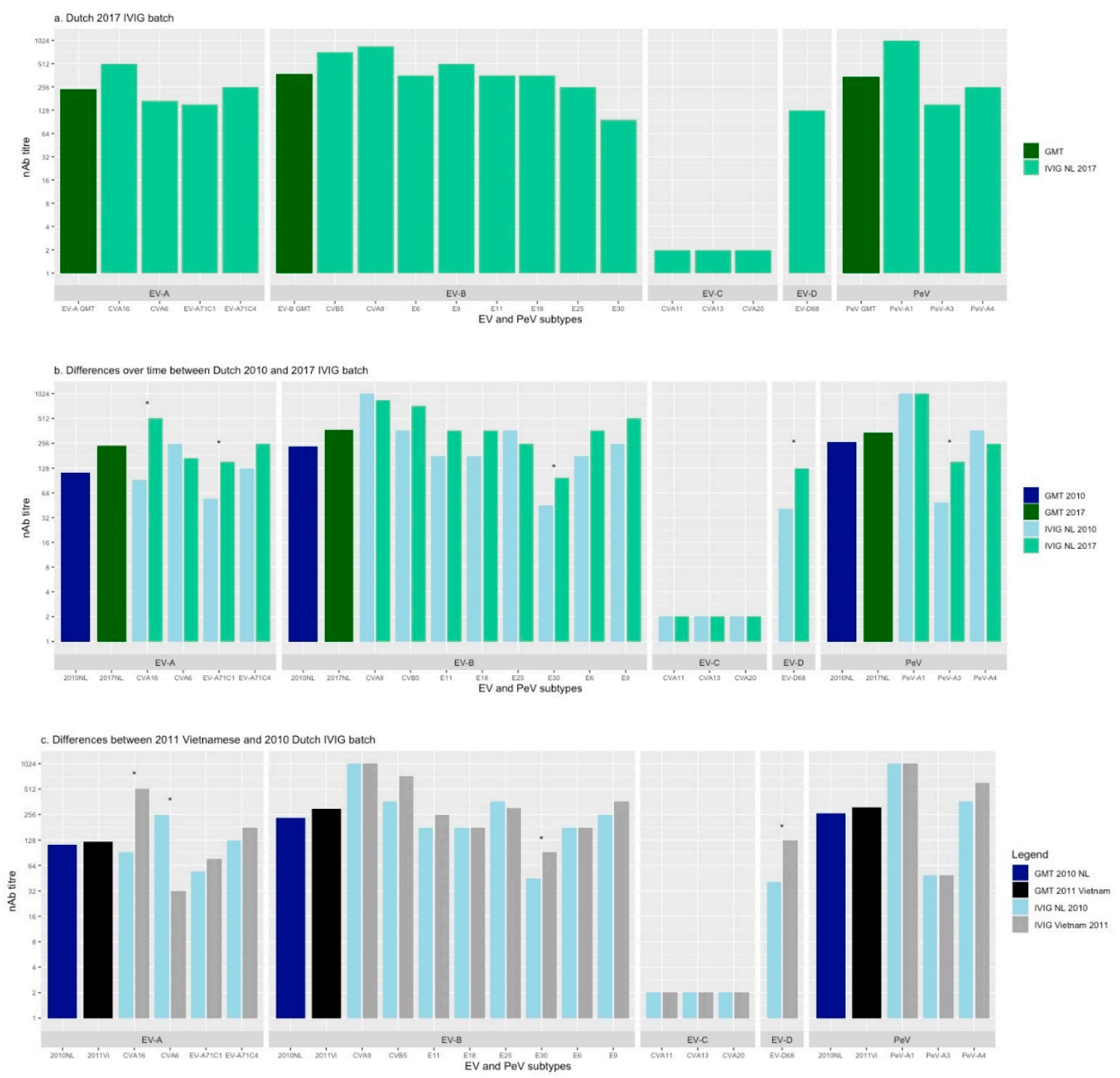

Figure 1. Neutralising antibody titres against different Entero- and Parechovirus strains a. Dutch 2017 IVIG batch b. Differences over time between Dutch 2010 and 2017 IVIG batch c. Differences between 2011 Vietnamese and 2010 Dutch IVIG batch. Asterisks $\left(^{*}\right)$ indicate a more than twofold difference in nAb titre between IVIG batches. GMT; geometric mean titre, IVIG; intravenous immunoglobulins, nAb titre; neutralising antibody titre, EV; enterovirus, PeV; parechovirus, CVA; coxsackievirus A, CVB; coxsackievirus B, E; echovirus, NL; the Netherlands, Vi; Vietnam.

\subsection{Neutralising Ab Titres Against EV and PeV in Dutch 2017 IVIG}

In the Dutch IVIG from 2017, the geometric mean nAb titres (GMT) were medium against the species Enterovirus A, Enterovirus $B$ and Parechovirus $A$ (1:241, 1:373 and 1:342, respectively) (Figure 1a). The nAb titres between types from Enterovirus $A$, Enterovirus $B$ and Parechovirus $A$ did not differ significantly $(p=0.275)$. No nAb titres $(<1: 4)$ were found against any of the types from Enterovirus $C$ species, including after chloroform treatment. The 2017 IVIG contained $\mathrm{nAb}$ titres against the three virus types belonging to Enterovirus $A$. A high nAb titre (1:512) was found against CVA16 and medium $\mathrm{nAb}$ titres were found against CVA6 and EV-A71. The 2017 IVIG contained nAb titres against all Enterovirus $B$ types tested. It showed low nAb titres against E30 (1:97), despite the high frequency of 
detection according to VIRO-Typened (Table 1 ). Neutralising Ab titres against other types of EV-B species ranged from medium (E6, E11, E25, E18) to high (E9, CVB5, CVA9). A nAb titre of 1:128 was found against EV-D68. PeV-A3 and PeV-A4 showed medium and high $\mathrm{nAb}$ titres, respectively. PeV-A1 had the highest nAb titre (1:1024).

\subsection{Neutralising Ab Titres over Time in Dutch IVIG}

In the Dutch IVIG batches from 2010 and 2017, the nAb titres against types from Enterovirus $A$, Enterovirus $B$ and Parechovirus $A$ did not differ significantly between the two years $(p<0.05)$ (Figure $1 b)$. Concerning the Enterovirus $A$ species the $\mathrm{nAb}$ titres against CVA16 and EV-A71C1 increased $>2$-fold over time (from 1:91 to 1:512 and from 1:54 to 1:152, respectively). The $\mathrm{nAb}$ titre against CVA6 tended to decrease. One Enterovirus B type, E30, showed an >2-fold increase between 2010 and 2017 (from 1:45 to 1:97). The highest nAb titre in 2010 and 2017 was found against CVA9 (1:1024 and 1:864, respectively). As for the Dutch 2017 batch, the included types from Enterovirus $C$ were not neutralised by the Dutch 2010 batch. The titre against EV-D68 increased $>2$-fold over time. The nAb titre against this type increased from 1:41 in 2010 to 1:128 in 2017. The nAb titre against PeV-A3 increased $>2$-fold from 1:49 to 1:152.

\subsection{Neutralising Ab Titres in 2010 Dutch and 2011 Vietnamese IVIG batch}

The mean $\mathrm{nAb}$ titres against types from Enterovirus A, Enterovirus B and Parechovirus A did not differ significantly between the Dutch 2010 and Vietnamese 2011 IVIG batch ( $p$ $<0.05)$. An important difference in the Enterovirus A types was the $>2$-fold lower $n A b$ titre against CVA6 (1:32 against 1:256, respectively) and $>2$-fold higher $n A b$ titre against CVA16 (1:512 against 1:91, respectively) in the Vietnamese in comparison to the Dutch IVIG batch. No differences between the two batches for the different EV-A71 genetic clades C1 (Dutch strain) and C4 (Japanese strain) were observed. Neutralising Ab titres against the included Enterovirus B types were overall comparable between the two batches. Similar to the Dutch IVIG batches, the included Enterovirus $C$ types were not neutralised by the Vietnamese batch. The nAb titres of the Vietnamese IVIG batch against PeV-A1, PeV-A3 and PeV-A4 were of the same size order as the Dutch IVIG batch.

\section{Discussion}

In our study, we used IVIG as a representative of the immunological profile of a population and investigated sero-prevalence of NPEV and PeV in IVIG batches from 2010, 2011 and 2017 from the Netherlands and Vietnam.

The sero-prevalence in the Dutch IVIG batches was comparable to the VIRO-Typened geno-surveillance data of NPEV and PeV, with overall medium nAb titres present in IVIG against included Enterovirus $A$, Enterovirus $B$ and Parechovirus $A$ types. The GMT of included Enterovirus $B$ species types was the highest, which corresponds to the fact that Enterovirus B members are the most commonly isolated types in VIRO-Typened [10]. No neutralisation of any included Enterovirus $C$ type was observed. Types belonging to Enterovirus $C$ species are rarely reported according to VIRO-Typened $[10,15,16]$. Our data suggest an absence of population immunity against these types. However, it is possible that the included Enterovirus $C$ types, do not reflect circulating strains, as they were isolated in 1988. The Dutch IVIG batches not only neutralised the Dutch EV-A71C1 strain but also the Japanese EV-A71C4 strain. Indeed, in a study from van der Sanden et al., intra-typic cross-neutralisation was reported for some but not all EV-A71 genotypes (including EV$\mathrm{A} 71 \mathrm{C} 1$ and EV-A71C4) [6]. Another striking observation was the low $\mathrm{nAb}$ titre against EV-D68 in 2010, which increased in 2017. EV-D68 was rarely detected before 2010 when it started causing large outbreaks worldwide, the same year it had its first upsurge in VIRO-Typened $[10,17]$. In contrast, other research groups reported high nAb titres against a recent clinical EV-D68 B3 strain from 2016 in serum samples before and after the first reported upsurge in 2010 [18,19]. 
In contrast to our findings, a previous study from 1997 failed to show a correlation between $\mathrm{nAb}$ titres in two Dutch IVIG batches and the prevalence of certain serotypes. However, at that time surveillance was based on virus culture and serotyping, which is less sensitive than genotyping methods. Furthermore, mostly reference strains were used instead of current circulating strains [8].

Against some types we found unexpectedly low levels of nAb titres. While E30 is known to circulate endemically in the Netherlands and to cause large outbreaks in Vietnam $[10,13,15,20,21]$, low nAb titres were found in all IVIG batches. It is possible, however, that other genetic clades are more dominant or of equally importance than the strains we included [22]. We found low nAb titres against CVA6 in the Vietnamese IVIG batch, while the last decade CVA6 has replaced CVA16 and EVA71 as the primary cause of HFMD in several Asian-Pasific countries [2]. It could be, however, that more recent IVIG batches from Vietnam would show higher levels of nAb titres against CVA6. Unfortunately, we did not have access to IVIG from Vietnam after 2011. Low nAb titres against PeV-A3 were detect in the 2010 Dutch IVIG batch. Other studies have shown high population seropositivity against PeV-A3 in the Netherlands but declining seropostivity in age groups over 30 could suggest that widespread circulation has emerged only recently $[13,15,23]$. In fact, $\mathrm{nAb}$ titres against PeV-A3 did increase in the 2017 Dutch IVIG batch. Low nAb titres against PeV-A3 were also found in the 2011 Vietnamese IVIG batch, where epidemics are known to occur every 2-3 years since 2006 [24]. Our study did not include a more recent Vietnamese IVIG batch, so we were unable to evaluate if $\mathrm{nAb}$ titres against $\mathrm{PeV}-\mathrm{A} 3$ also increase over time in IVIG from Vietnam.

Our study had several strengths. We measured nAb titres against members from all NPEV and Parechovirus A species which gave a broad footprint. Furthermore, strains were selected to reflect as much as possible current circulating types. However, except for EVA71, we used only a single clinical strain for each type. In general, it is assumed that crossneutralisation is limited between different types [1]. Recent studies, however, did show differences in the level of intra-typic cross-neutralisation between different clades [6,25]. Therefore, we cannot differentiate between neutralisation and cross-neutralisation to other genetic (sub)types. As different genetic (sub)types can circulate and vary between regions and over time, it could explain some of the differences we found and appropriate strains have to be selected adapted to the time period and geographical region of the IVIG. Another limitation is that not all age groups were included in our study, as IVIG do not contain plasma from children. The incidence of $\mathrm{EV}$ and $\mathrm{PeV}$ infections is the highest in this age group and $\mathrm{nAb}$ measured in IVIG can be a reflection of infections encountered many years ago. This interval is important to consider when analysing changes over time. Finally, we cannot exclude an effect of different cell lines on $\mathrm{nAb}$ titres. In conclusion, we demonstrated that IVIG can be a valuable and reliable tool to generate sero-prevalence data. Moreover, it can be used to evaluate changes over time and to expose population susceptibility to certain (sub)types. Therefore, it could be an important tool, in addition to geno-surveillance data, in predicting future upsurges and outbreaks by upcoming geno-subtypes. Furthermore, it has the potential to provide data on circulating strains causing more mild disease. These types could be missed by geno-surveillance which is mostly based on sequence typing of $\mathrm{EV}$ and PeV found in clinical samples. As the Enterovirus $C$ strains we studied, were isolated in 1988, more studies are needed to elucidate circulation and population immunity against Enterovirus $C$ species. Finally, to be able to compare data generated by different sero-prevalence studies, reference strains and techniques should be further harmonised.

Supplementary Materials: The following are available online at https: / www.mdpi.com/article / 10.3390/v13061028/s1, Table S1: Selected Entero- and Parechovirus strain characteristics, Table S2: Neutralising antibody titres against the different Entero- and Parechovirus strains in the different IVIG batches.

Author Contributions: Conceptualization, K.C.W. and D.P.; methodology, K.C.W., K.v.d.S., H.v.E.; formal analysis, K.v.d.S., K.C., H.v.E., G.K.; data curation, K.v.d.S.; writing—original draft preparation, 
K.C.; writing-review and editing, K.C.W., J.-L.M., K.v.d.S., L.B.; visualization, K.C.; supervision, J.-L.M., K.C.W., D.P.; project administration, H.v.E., G.K.; All authors have read and agreed to the published version of the manuscript.

Funding: Katja Wolthers and Dasja Pajkrt are funded by EU H2020 ITN grant number 812673 (OrganoVIR).

Institutional Review Board Statement: Not applicable.

Informed Consent Statement: Not applicable.

Data Availability Statement: The data presented in this study are available in Supplementary Table S2.

Acknowledgments: We would like to thank Kim Benschop from National Institute of Public Health and Environment (RIVM) for sharing VIRO-Typened data.

Conflicts of Interest: The authors declare no conflict of interest. The funders had no role in the design of the study; in the collection, analyses, or interpretation of data; in the writing of the manuscript, or in the decision to publish the results.

\section{References}

1. Anastasina, M.; Domanska, A.; Palm, K.; Butcher, S. Human picornaviruses associated with neurological diseases and their neutralization by antibodies. J. Gen. Virol. 2017, 98, 1145-1158. [CrossRef] [PubMed]

2. Pons-Salort, M.; Parker, E.P.K.; Grassly, N.C. The epidemiology of non-polio enteroviruses: Recent Advances and Outstanding Questions. Curr. Opin. Infect. Dis. 2015, 28, 479-487. [CrossRef] [PubMed]

3. De Crom, S.C.M.; Rossen, J.W.A.; Van Furth, A.M.; Obihara, C.C. Enterovirus and parechovirus infection in children: A brief overview. Eur. J. Nucl. Med. Mol. Imaging 2016, 175, 1023-1029. [CrossRef] [PubMed]

4. Rantakallio, P.; Leskinen, M.; Von Wendt, L. Incidence and Prognosis of Central Nervous System Infections in a Birth Cohort of 12,000 Children. Scand. J. Infect. Dis. 1986, 18, 287-294. [CrossRef]

5. Vollbach, S.; Müller, A.; Drexler, J.F.; Simon, A.; Drosten, C.; Eis-Hübinger, A.M.; Panning, M. Prevalence, type and concentration of human enterovirus and parechovirus in cerebrospinal fluid samples of pediatric patients over a 10-year period: A retrospective study. Virol. J. 2015, 12, 1-6. [CrossRef]

6. Van Der Sanden, S.M.; Koen, G.; Van Eijk, H.; Koekkoek, S.M.; De Jong, M.D.; Wolthers, K.C. Prediction of Protection against Asian Enterovirus 71 Outbreak Strains by Cross-neutralizing Capacity of Serum from Dutch Donors, The Netherlands. Emerg. Infect. Dis. 2016, 22, 1562-1569. [CrossRef]

7. Pons-Salort, M.; Grassly, N.C. Serotype-specific immunity explains the incidence of diseases caused by human enteroviruses. Science 2018, 361, 800-803. [CrossRef] [PubMed]

8. Galama, J.M.; Vogels, M.T.; Jansen, G.H.; Gielen, M.; Heessen, F.W. Antibodies against enteroviruses in intravenous Ig preparations: Great variation in titres and poor correlation with the incidence of circulating serotypes. J. Med. Virol. 1997, 53, 273-276. [CrossRef]

9. Wildenbeest, J.G.; Broek, P.J.V.D.; Benschop, K.S.; Koen, G.; Wierenga, P.C.; Vossen, A.C.; Kuijpers, T.W.; Wolthers, K.C. Pleconaril revisited: Clinical course of chronic enteroviral meningoencephalitis after treatment correlates with in vitro susceptibility. Antivir. Ther. 2011, 17, 459-466. [CrossRef] [PubMed]

10. Benschop, K.S.M.; Rahamat-Langendoen, J.C.; Van Der Avoort, H.G.A.M.; Claas, E.C.J.; Pas, S.D.; Schuurman, R.; Verweij, J.J.; Wolthers, K.C.; Niesters, H.G.M.; Koopmans, M.P.G.; et al. VIRO-TypeNed, systematic molecular surveillance of enteroviruses in the Netherlands between 2010 and 2014. Eurosurveillance 2016, 21. [CrossRef]

11. Kapsenberg, J.G.; Ras, A.; Korte, J. Improvement of Enterovirus Neutralization by Treatment with Sodium Deoxycholate or Chloroform. Intervirology 1979, 12, 329-334. [CrossRef]

12. Karelehto, E.; Van Der Sanden, S.; Geraets, J.A.; Domanska, A.; Van Der Linden, L.; Hoogendoorn, D.; Koen, G.; Van Eijk, H.; Shakeel, S.; Beaumont, T.; et al. Strain-dependent neutralization reveals antigenic variation of human parechovirus 3. Sci. Rep. 2017, 7, 1-10. [CrossRef] [PubMed]

13. Karelehto, E.; Brouwer, L.; Benschop, K.; Kok, J.; Basile, K.; McMullan, B.; Rawlinson, W.; Druce, J.; Nicholson, S.; Selvarangan, R.; et al. Seroepidemiology of Parechovirus A3 Neutralizing Antibodies, Australia, the Netherlands, and United States. Emerg. Infect. Dis. 2019, 25, 148-152. [CrossRef] [PubMed]

14. Zhu, R.; Cheng, T.; Yin, Z.; Liu, D.; Xu, L.; Li, Y.; Wang, W.; Liu, J.; Que, Y.; Ye, X.; et al. Serological survey of neutralizing antibodies to eight major enteroviruses among healthy population. Emerg. Microbes Infect. 2018, 7, 1-15. [CrossRef]

15. Van Der Sanden, S.M.G.; Koopmans, M.P.G.; Van Der Avoort, H.G.A.M. Detection of human enteroviruses and parechoviruses as part of the national enterovirus surveillance in the Netherlands, 1996-2011. Eur. J. Clin. Microbiol. Infect. Dis. 2013, 32, $1525-1531$. [CrossRef] [PubMed]

16. Janes, V.A.; Minnaar, R.; Koen, G.; Van Eijk, H.; Haan, K.D.-D.; Pajkrt, D.; Wolthers, K.C.; Benschop, K.S. Presence of human nonpolio enterovirus and parechovirus genotypes in an Amsterdam hospital in 2007 to 2011 compared to national and international published surveillance data: A comprehensive review. Eurosurveillance 2014, 19. [CrossRef] [PubMed] 
17. Meijer, A.; van der Sanden, S.; Snijders, B.E.; Jaramillo-Gutierrez, G.; Bont, L.; van der Ent, C.K.; Overduin, P.; Jenny, S.L.; Jusic, E.; van der Avoort, H.G.; et al. Emergence and epidemic occurrence of enterovirus 68 respiratory infections in The Netherlands in 2010. Virology 2012, 423, 49-57. [CrossRef]

18. Karelehto, E.; Koen, G.; Benschop, K.; Van Der Klis, F.; Pajkrt, D.; Wolthers, K. Enterovirus D68 serosurvey: Evidence for endemic circulation in the Netherlands, 2006 to 2016. Eurosurveillance 2019, 24, 1800671. [CrossRef]

19. Kamau, E.; Harvala, H.; Blomqvist, S.; Nguyen, D.; Horby, P.; Pebody, R.; Simmonds, P. Increase in Enterovirus D68 Infections in Young Children, United Kingdom, 2006-2016. Emerg. Infect. Dis. 2019, 25, 1200-1203. [CrossRef]

20. Takamatsu, Y.; Uchida, L.; Nga, P.T.; Okamoto, K.; Nabeshima, T.; Thao, D.T.; Hai do, T.; Tuyet, N.T.; Duc, H.M.; Luat le, X.; et al. An approach for differentiating echovirus 30 and Japanese encephalitis virus infections in acute meningitis/encephalitis: A retrospective study of 103 cases in Vietnam. Virol. J. 2013, 10, 280. [CrossRef]

21. Lee, J.Y.; Seo, Y.; Choi, U.Y.; Kim, J.-H.; Kang, J.H. Seroepidemiology of echovirus 30 in Korean children. World J. Pediatr. 2017, 13, 611-614. [CrossRef] [PubMed]

22. Benschop, K.S.; Broberg, E.K.; Hodcroft, E.; Schmitz, D.; Albert, J.; Baicus, A.; Bailly, J.-L.; Baldvinsdottir, G.; Berginc, N.; Blomqvist, S.; et al. Molecular Epidemiology and Evolutionary Trajectory of Emerging Echovirus 30, Europe. Emerg. Infect. Dis. 2021, 27, 1616-1626. [CrossRef] [PubMed]

23. Westerhuis, B.; Kolehmainen, P.; Benschop, K.; Nurminen, N.; Koen, G.; Koskiniemi, M.; Simell, O.; Knip, M.; Hyoty, H.; Wolthers, K.; et al. Human parechovirus seroprevalence in Finland and the Netherlands. J. Clin. Virol. 2013, 58, 211-215. [CrossRef]

24. Aizawa, Y.; Suzuki, Y.; Watanabe, K.; Oishi, T.; Saitoh, A. Clinical utility of serum samples for human parechovirus type 3 infection in neonates and young infants: The 2014 epidemic in Japan. J. Infect. 2016, 72, 223-232. [CrossRef] [PubMed]

25. Imamura, T.; Okamoto, M.; Nakakita, S.-I.; Suzuki, A.; Saito, M.; Tamaki, R.; Lupisan, S.; Roy, C.N.; Hiramatsu, H.; Sugawara, K.-E.; et al. Antigenic and Receptor Binding Properties of Enterovirus 68. J. Virol. 2014, 88, 2374-2384. [CrossRef] 